\title{
Formation of Nanoporous Tin Oxide Layers on Different Substrates during Anodic Oxidation in Oxalic Acid Electrolyte
}

\author{
Leszek Zaraska, ${ }^{1}$ Michał Bobruk, ${ }^{2}$ and Grzegorz D. Sulka ${ }^{1}$ \\ ${ }^{1}$ Department of Physical Chemistry and Electrochemistry, Faculty of Chemistry, Jagiellonian University in Kraków, \\ Ingardena 3, 30-060 Kraków, Poland \\ ${ }^{2}$ Faculty of Materials Science and Ceramics, AGH University of Science and Technology, Mickiewicza 30, 30-059 Kraków, Poland
}

Correspondence should be addressed to Leszek Zaraska; zaraska@chemia.uj.edu.pl

Received 6 May 2015; Accepted 14 June 2015

Academic Editor: Jan A. Jung

Copyright (C) 2015 Leszek Zaraska et al. This is an open access article distributed under the Creative Commons Attribution License, which permits unrestricted use, distribution, and reproduction in any medium, provided the original work is properly cited.

\begin{abstract}
Nanoporous tin oxide layers were obtained on various Sn substrates including high- and low-purity foils and wire by one-step anodic oxidation carried out in a $0.3 \mathrm{M}$ oxalic acid electrolyte at various anodizing potentials. In general, amorphous oxide layers with the atomic ratio of $\mathrm{Sn}: \mathrm{O}(1: 1)$ were grown during anodization, and a typical structure of the as-obtained film consists of the "outer" layer with less regular, interconnetted pores and the "inner" layer with much more uniform and regular channels formed as a result of vigorous gas evolution. It was found that the use of electrochemical cell with the sample placed horizontally on the metallic support and stabilized by the Teflon cover, instead of the typical two-electrode system with vertically arranged electrodes, can affect the morphology of as-obtained layers and allows fabrication of nanoporous oxides even at anodizing potentials up to $11 \mathrm{~V}$. An average pore diameter in the "outer" oxide layer increases with increasing anodizing potential, and no significant effect of substrate purity on the structure of anodic film was proved, except better uniformity of the oxides grown on high-purity Sn. A strong linear relationship between the average steady-state current density and anodizing potential was also observed.
\end{abstract}

\section{Introduction}

During the recent years, $\mathrm{SnO}_{2}$ based materials have become of growing interest due to their promising electronic, optical, and photoelectrochemical properties and wide potential applications, for example, in solid-state gas sensors [1-3], solar cells [4], and catalysts [3]. In addition, tin oxide is a very promising alternative to the commercial graphite anode in Li-ion batteries offering much higher specific capacity [5]. The use of nanostructured oxides instead of bulk $\mathrm{SnO}_{2}$ can dramatically improve its functionality and properties, mainly due to the much higher surface areas and restricted sizes [6]. Many different strategies for fabrication of $\mathrm{SnO}_{2}$ nanomaterials have been already proposed including thermal evaporation $[7,8]$, hydrothermal methods [9], template assisted procedures [10], electrospinning [11], and electrochemical methods [12]. Among them, anodic oxidation (anodization), proposed originally by Shin et al. in 2004 , seems to be a very interesting, simple, and cost-effective strategy for the fabrication of nanoporous tin oxide-based layers on the surface of metallic tin [13]. It is well known that anodic oxidation of tin in acidic (e.g., $\mathrm{H}_{2} \mathrm{C}_{2} \mathrm{O}_{4}$ ) [1320], alkaline (e.g., $\mathrm{NaOH}[21,22], \mathrm{NH}_{3}$ [23]) solutions or sulfide and fluoride-based electrolytes [24, 25] can result in the formation of nanoporous oxide layers, and in most cases, nanopores are randomly distributed across the surface. The morphology of nanoporous oxide is completely different from the structures observed in anodic alumina or titania $[26,27]$. The nanochannels, almost perpendicular to the surface, are rather discontinues, and the oxide layer often consists of stacked layers of several hundreds of nanometers $[13,19]$. Recently, Feng et al. reported that anodic oxidation of tin in dimethyl sulfoxide (DMSO)/water mixtures containing citric acid can lead to the formation of nanostructured citrate 
(a)

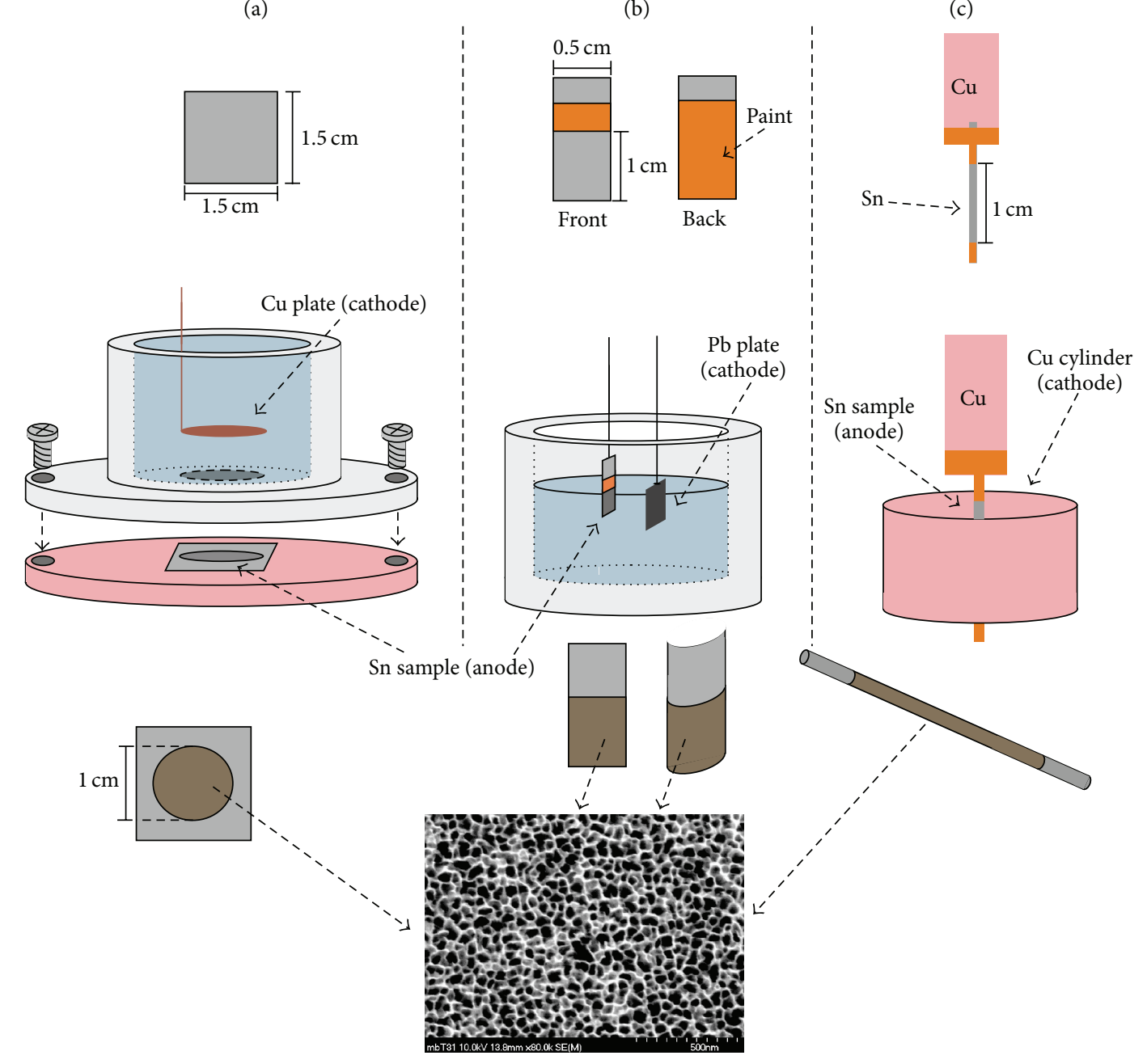

(b) (c)

FIGURE 1: Schematic representation of experimental setups used for anodizing of $S n$ foils $(a, b)$ and $S n$ wire (c). complexes that can be converted to polycrystalline $\mathrm{SnO}_{2}$ nanostrips with a porous structure [28]. The use of electrolyte without DMSO results also in the formation of nanoporous oxide layers [28].

According to recent works, anodically grown nanoporous tin oxides can offer many promising applications, for example, in solid-state gas sensors [15, 24, 29], lithium-ion batteries [30-33], solar cells [23], supercapacitors [34], and photonic crystals [20].

It should be mentioned that a further application of nanoporous tin oxide depends on the morphology, composition, and structure of the oxide layer which are strongly affected by anodizing conditions such as applied potential, kind and concentration of electrolyte, temperature, purity and shape of the substrate, or even electrochemical cell geometry. Therefore, to achieve the precise control of the oxide structure formed during anodic oxidation, a detailed correlation between the morphology of anodic oxide layers and anodizing conditions is required.

In our recent work, we discussed the effect of anodizing conditions on the formation and structure of anodic tin oxides formed during anodization of low-purity $\mathrm{Sn}$ foil in a conventional electrochemical cell with vertically aligned electrodes [35]. Here, we focus mainly on the effect of different cell geometry on the growth and morphology of nanoporous tin oxide layers and on possibilities for fabricating anodic oxides on various $\mathrm{Sn}$ substrates.

\section{Materials and Methods}

Low-purity (98.8\%) and high-purity (99.999\%) Sn foils (Goodfellow, $0.5 \mathrm{~mm}$ in thickness) as well as $\mathrm{Sn}$ wire (99.999\%) (Sigma Aldrich, $0.5 \mathrm{~mm}$ in diameter) were used as starting materials for anodization. Prior to anodizing, the samples with specified dimensions (see Figure 1) were cleaned by degreasing in ethanol and then acetone and dried. In some cases, the surface area of the sample was defined by using an acid resistant paint (see Figures 1(b) and 1(c)). Two different electrochemical cells were used for anodization of the Sn foils: a homemade Teflon cell (volume of $100 \mathrm{~cm}^{3}$ ) with the sample placed horizontally on the metallic plate (A-type cell, Figure 1(a)) and a conventional electrochemical cell with 


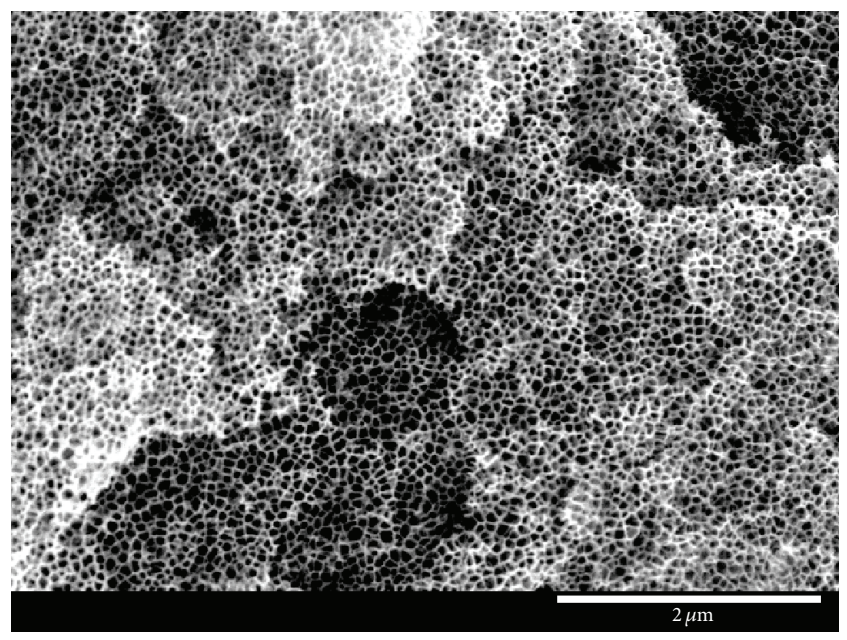

(a)

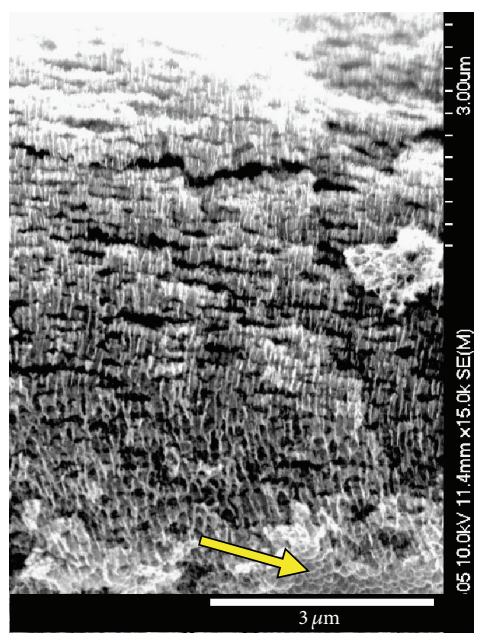

(b)

Figure 2: FE-SEM top-view (a) and cross-sectional view (b) images of nanoporous oxide layer obtained during one-step anodization of high-purity $\mathrm{Sn}$ in $0.3 \mathrm{M}$ oxalic acid at $9 \mathrm{~V}$. Duration of the process was $1800 \mathrm{~s}$. The arrow in (b) indicates the bottom of the oxide layer.

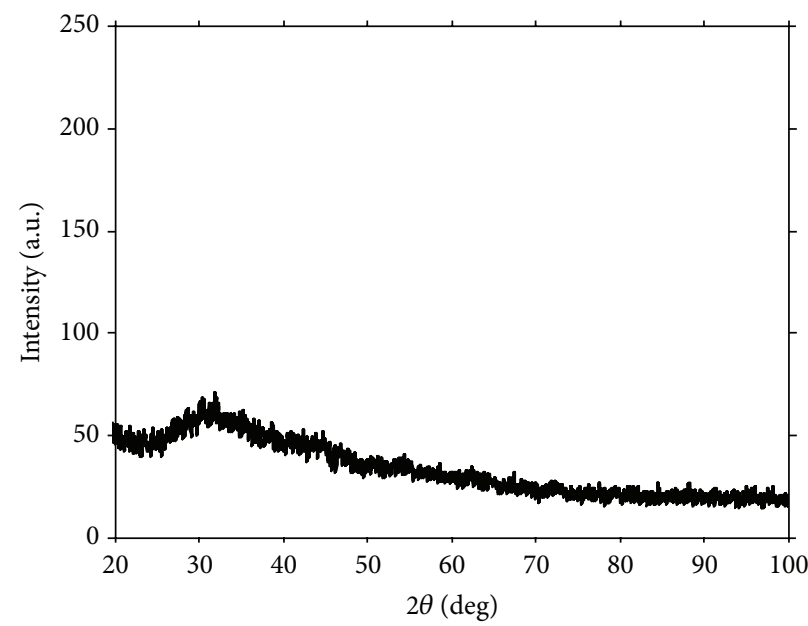

(a)

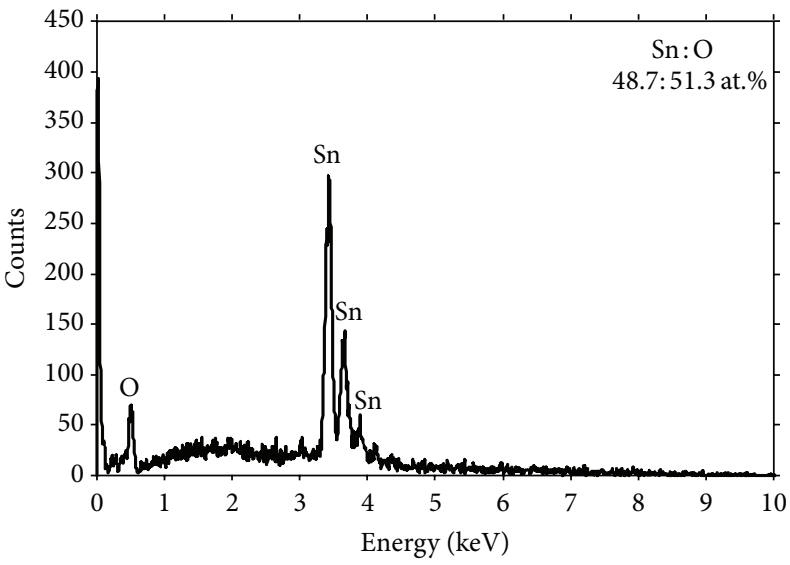

(b)

FIGURE 3: XRD pattern (a) and EDS spectrum (b) of the anodic tin oxide layer obtained during one-step anodization of low-purity Sn in B-type cell at a potential of $8 \mathrm{~V}$.

vertically aligned electrodes (B-type cell, Figure 1(b)). On the contrary, a different experimental setup with a cylindrical $\mathrm{Cu}$ cathode was used for the Sn wire anodizing (see Figure 1(c)). All anodization processes were carried out in two-electrode systems powered by the conventional DC power supply (Array 3646A), and current versus time curves were recorded by using the APPA207 multimeter. The porous anodic tin oxide layers were obtained by simple, potentiostatic one-step anodization processes in a $0.3 \mathrm{M}$ oxalic acid electrolyte under various operating conditions at room temperature. Just after anodizing, samples were rinsed several times with deionized water and then ethanol and dried.

The morphology of as-obtained oxide layers was analyzed by using a Field Emission Scanning Electron Microscope (FESEM/EDS, Hitachi S-4700 with a Noran System 7), and the composition of the anodic products was confirmed by energy dispersive X-ray spectroscopy (EDS) and X-ray diffraction (XRD) measurements. Structural features of nanoporous tin oxides were determined directly from FE-SEM images.

\section{Results and Discussion}

In general, anodic oxidation of all Sn substrates in oxalic acid electrolyte resulted in the formation of nanoporous oxide films on the metal surface. The FE-SEM images of typical structure of anodic layer after $1800 \mathrm{~s}$ of anodization of the high-purity Sn foil in the B-type cell at the potential of $9 \mathrm{~V}$ are shown in Figure 2. As can be seen, a completely irregular porous structure with randomly distributed nanochannels was formed during anodization (see Figure 2(a)). The typical 


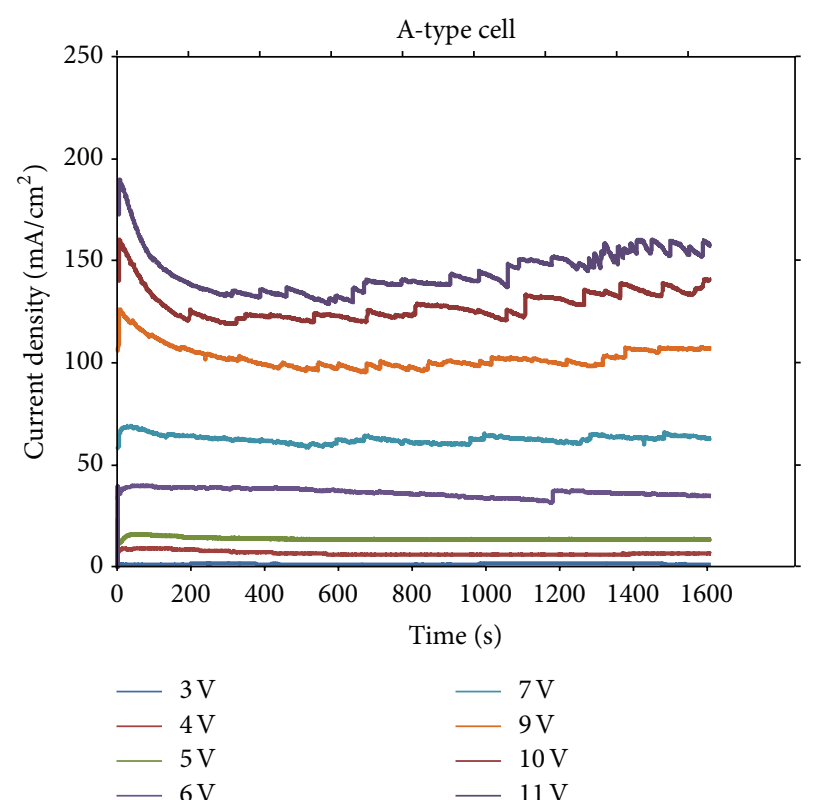

(a)

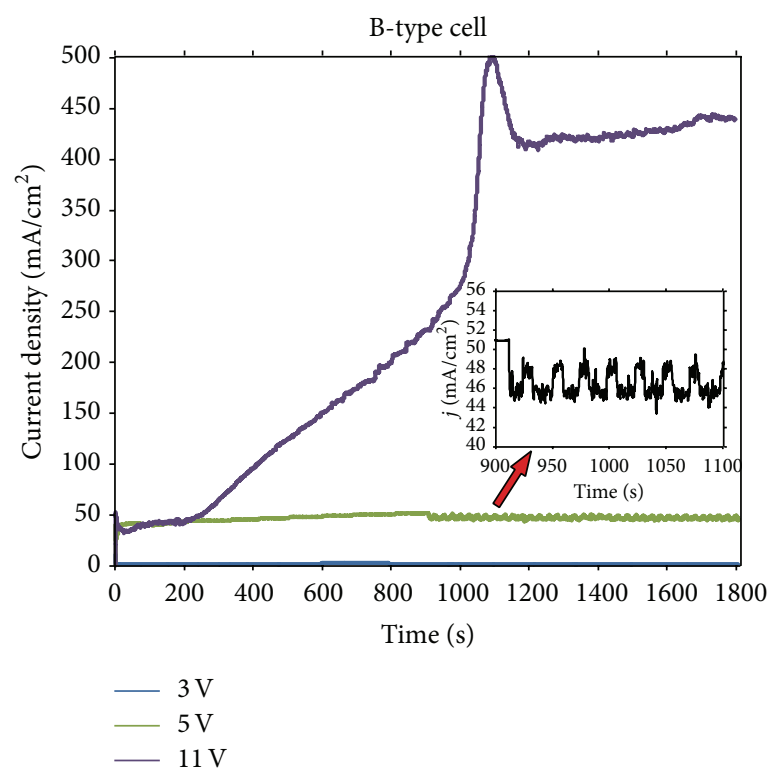

(b)

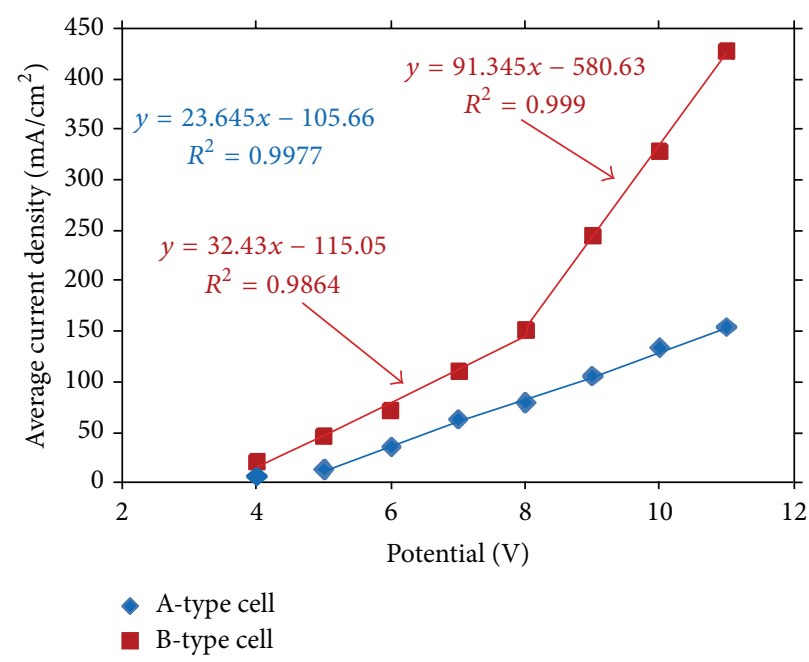

(c)

FiguRE 4: Current density versus time curves recorded during anodization of low-purity $\mathrm{Sn}$ in the A-type (a) and B-type cells (b) at various anodizing potentials together with the dependence between an average steady-state current density and anodizing potential (c).

layered oxide structure with a lot of internal gaps and transversal pores can be recognized in Figure 2(b).

The formation of such structures, being a result of a vigorous gas generation and evolution of the oxide morphology during anodizing in oxalic acid, was described and discussed in detail in our previous works [19, 35]. It should be mentioned that as-obtained anodic tin oxide was amorphous (see XRD spectrum with no significant peaks, Figure 3(a)). Moreover, the dark color of oxide and the atomic ratio of $\mathrm{Sn}$ : O obtained from the EDS analysis (1:1) (Figure 3(b)) suggest that amorphous stannous oxide $(\mathrm{SnO})$ could be initially formed as a result of anodic oxidation of metallic tin in oxalic acid electrolyte. However, according to the recent works the nature of as-anodized tin oxide still remains uncertain [25]. The as-obtained amorphous product can be converted to crystalline $\mathrm{SnO}_{2}$ by further annealing at temperatures higher than $500^{\circ} \mathrm{C}$. Nonetheless, a relatively low melting point of metallic $\mathrm{Sn}\left(\sim 230^{\circ} \mathrm{C}\right)$ significantly hinders the possibility of formation of crystalline $\mathrm{SnO}_{2}$ on the $\mathrm{Sn}$ support.

The current density versus time curves recorded during anodization of low-purity $\mathrm{Sn}$ in the A-type cell at various potentials in $0.3 \mathrm{M}$ oxalic acid is shown in Figure 4(a). A significant increase in current density with increasing anodizing potential can be easily recognized. This trend is 

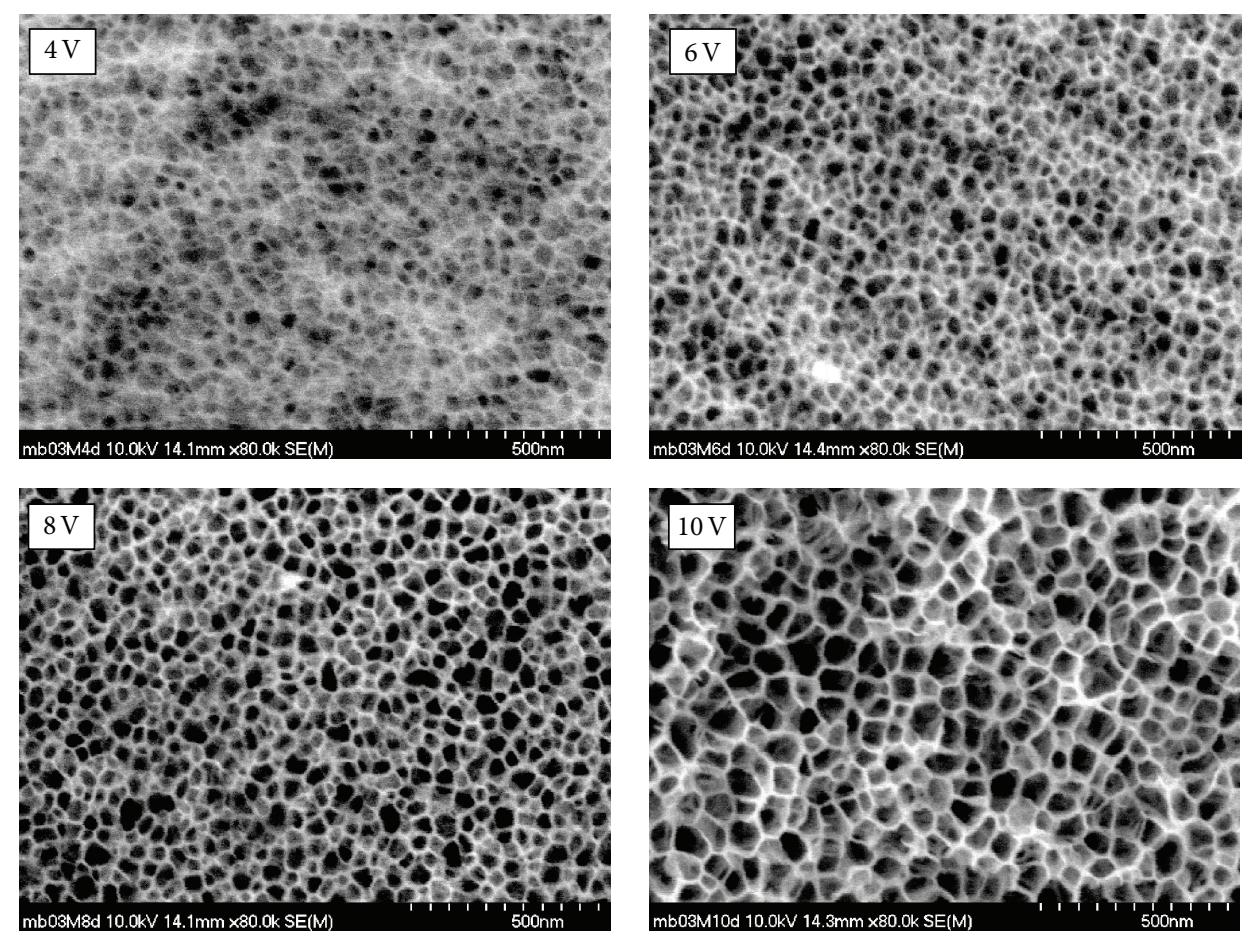

FIGURE 5: FE-SEM images of nanoporous tin oxide layers formed by one-step anodization in $0.3 \mathrm{M}$ oxalic acid under different anodizing conditions. The A-type electrochemical cell was used.

in general agreement with that observed for anodizing of the same kind of substrate in the B-type cell, reported in our recent work [35]. However, some meaningful differences in current-time behavior were observed when cell geometry was changed. Firstly, the values of an average steady-state current density recorded during anodization in the A-type cell were considerably lower from that observed during anodizing in the B-type system (compare blue and red lines in Figure 4(c)). Moreover, the higher the anodizing potential is applied, the greater the difference between the average steady-state current density values is. This phenomenon can be attributed to the fact that when anodization was carried out in the B-type cell with vertically arranged electrodes, a characteristic deformation of the sample (see Figure 1(b)) and deterioration of the oxide layer were observed as a consequence of high volume expansion of the oxide layer and a low mechanical strength of the pliant Sn foil. This leads to a significant increase of the active surface area of the anode during anodization and continuous growth of the anodic current density during electrolysis. It was observed especially for quite long processes ( $>10 \mathrm{~min}$ ) and at relatively high anodizing potentials. The use of the A-type cell with the sample placed horizontally on the metallic support and stabilized by the Teflon cover (see Figure 1(a)) prevents the Sn foil from a mechanical deformation and changing its shape.

In addition, quite different anodic tin behavior at higher anodizing potentials $(>9)$ was observed when the A-type electrochemical cell was used instead of the B-type one.
When the configuration with vertically aligned electrodes was used, an increase in the anodizing potential above $9 \mathrm{~V}$ resulted in the initial formation of the quite thick, passive layer on the electrode surface. The process was accompanied by a rapid decrease in the recorded current density (see the current density versus time curve recorded in the Btype cell during anodization at $11 \mathrm{~V}$, Figure 4(b)). After a certain time, the breakdown of the passive layer occurs causing a significant increase of current. A further continued anodization leads to formation of the porous oxide structure [35]. When the A-type cell was used, no initial formation of the passive layer on the low-purity Sn substrate was observed, even at anodizing potentials up to $11 \mathrm{~V}$ (see Figure 4(a)).

A strong linear relationship between the average current density and applied potential, similar to that reported in our previous work [35], was observed (see Figure 4(c)). This suggests that the reaction is limited by mass transfer in the electrolyte. It should be mentioned that the similar linear relationships between current density and anodizing potential were observed by Wang et al. [21] and by Ono and Asoh [36] for anodization carried out in various acidic and alkaline electrolytes.

In some cases, when anodization processes were carried out in the B-type cell, spontaneous periodical current oscillations analogous to those recorded by Wang et al. for alkaline electrolytes [22] were observed (see the current density versus time curve recorded during anodization at $5 \mathrm{~V}$, Figure 4(b)). This phenomenon can be explained in terms of the periodic oxygen gas bubbles formation and release from the Sn anode 


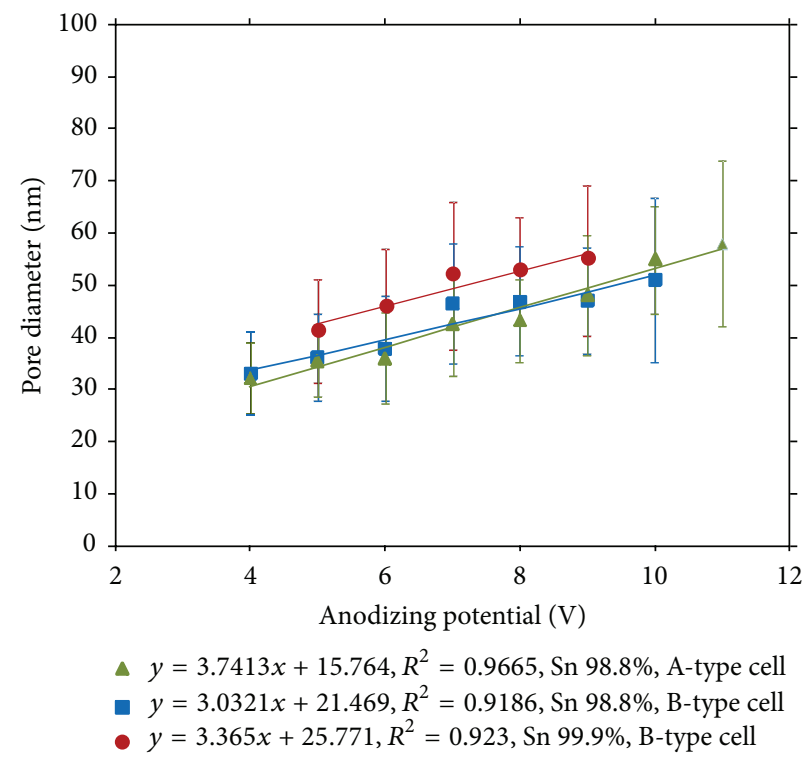

FIGURE 6: Average pore diameter as a function of anodizing potential.

that causes periodic redistribution of ion concentration in the electrolyte [22].

The FE-SEM top-view images of nanoporous tin oxide layers after $1800 \mathrm{~s}$ of anodization in $0.3 \mathrm{M}$ oxalic acid in the A-type cell under various anodizing potentials are shown in Figure 5 . As can be seen, an anodizing potential significantly affects the morphology of an outer oxide layer, and in general the higher potential is applied, the more open porous structure is formed. To confirm this quantitatively, an average pore diameter was estimated from the FE-SEM images (see Figure 6). The similar dependence was obtained by Chen et al. for anodization of high-purity Sn foils [16] and also by other groups for anodization of Sn electrochemically deposited on $\mathrm{Cu}$ [19] and low-purity Sn [35] (both in the B-type cell). As can be seen in Figure 6, no significant effect of cell geometry on the degree of pore opening in an outer oxide layer was found.

In order to compare the morphology of anodic tin layers grown on low-purity Sn with those obtained on high-purity Sn, a series of anodization processes of the high-purity substrate was also performed in $0.3 \mathrm{M}$ oxalic acid at various operating conditions. Surprisingly, no important effect of substrate purity on the structure of anodic film was proved, except better uniformity of the oxides grown on high-purity $\mathrm{Sn}$. The FE-SEM images of anodic porous oxides formed during $1800 \mathrm{~s}$ anodizing of the pure $\mathrm{Sn}$ foil at $6 \mathrm{~V}$ and $7 \mathrm{~V}$ are shown in Figures $7(\mathrm{a})-7(\mathrm{c})$ and $7(\mathrm{f})-7(\mathrm{~h})$, respectively. A large area of the quite uniform, porous oxide film can be easily recognized in Figures 7(a) and 7(f). The only cracks in the oxide structure are located along the grain boundaries (see low magnification images, Figures 7(c) and 7(h)). On the contrary, the anodic oxide layers grown on low-purity $\mathrm{Sn}$ exhibited much more cracks and defects (see Figures $7(\mathrm{e})$ and $7(j))$, mainly due to smaller grain sizes in the starting material, and so the mechanical disintegration of oxide occurs faster and more easily. In addition, the presence of alloying elements in a low-purity substrate influences the local rate of oxide growth and disturbs the direction of electric field during anodization. As a result, a less uniform oxide layer with number of cracks and voids is formed (see Figures 7(d) and $7(i)$ ). It should be mentioned that slightly higher average pore diameter values were observed in the outer oxide layers grown on high-purity Sn (see Figure 6, red line); however, the degree of pore opening was still dependent on the potential applied during electrolysis.

Anodic tin oxide layers were grown also on the surface of Sn wire. The FE-SEM images of the surface of this kind of substrate before and after $30 \mathrm{~min}$ of anodizing at a potential of $5 \mathrm{~V}$ are shown in Figures 8(a) and 8(b), respectively. As can be seen in Figure 8(b), the anodic oxidation carried out in a $0.3 \mathrm{M}$ oxalic acid electrolyte resulted in the formation of the quite uniform tin oxide layer on the surface of Sn wire. Some cracks along the grain boundaries are noticeable; however, under optimized conditions, when anodizing potential is not too high and the process duration is not too long, the asobtained oxide layer is rather mechanically stable and not easily removable from the substrate. As can be seen in Figures $8(\mathrm{c})-8(\mathrm{f})$, the structure of the oxide layer grown on the surface of $\mathrm{Sn}$ wire is almost identical to that obtained on Sn foils. A dense array of irregular pores with the average diameter of about $40 \mathrm{~nm}$ is shown in Figure 8(c). As it is shown in Figure 8(d), a compact "barrier layer" at the bottoms of nanochannels, analogous to that observed in other anodic oxides (e.g., $\mathrm{Al}$ and $\mathrm{Ti}$ ), is formed during anodization. The cross-sectional views shown in Figures 8(e) and 8(f) indicate that the oxide layers grown on $\mathrm{Sn}$ wire are quite uniform; however, Figure 8(f) suggests also the formation of stack-like structures, similar to that synthesized electrochemically on Sn foils. It should be mentioned that the existence of this kind of additional transversal cracks and voids strongly affects 

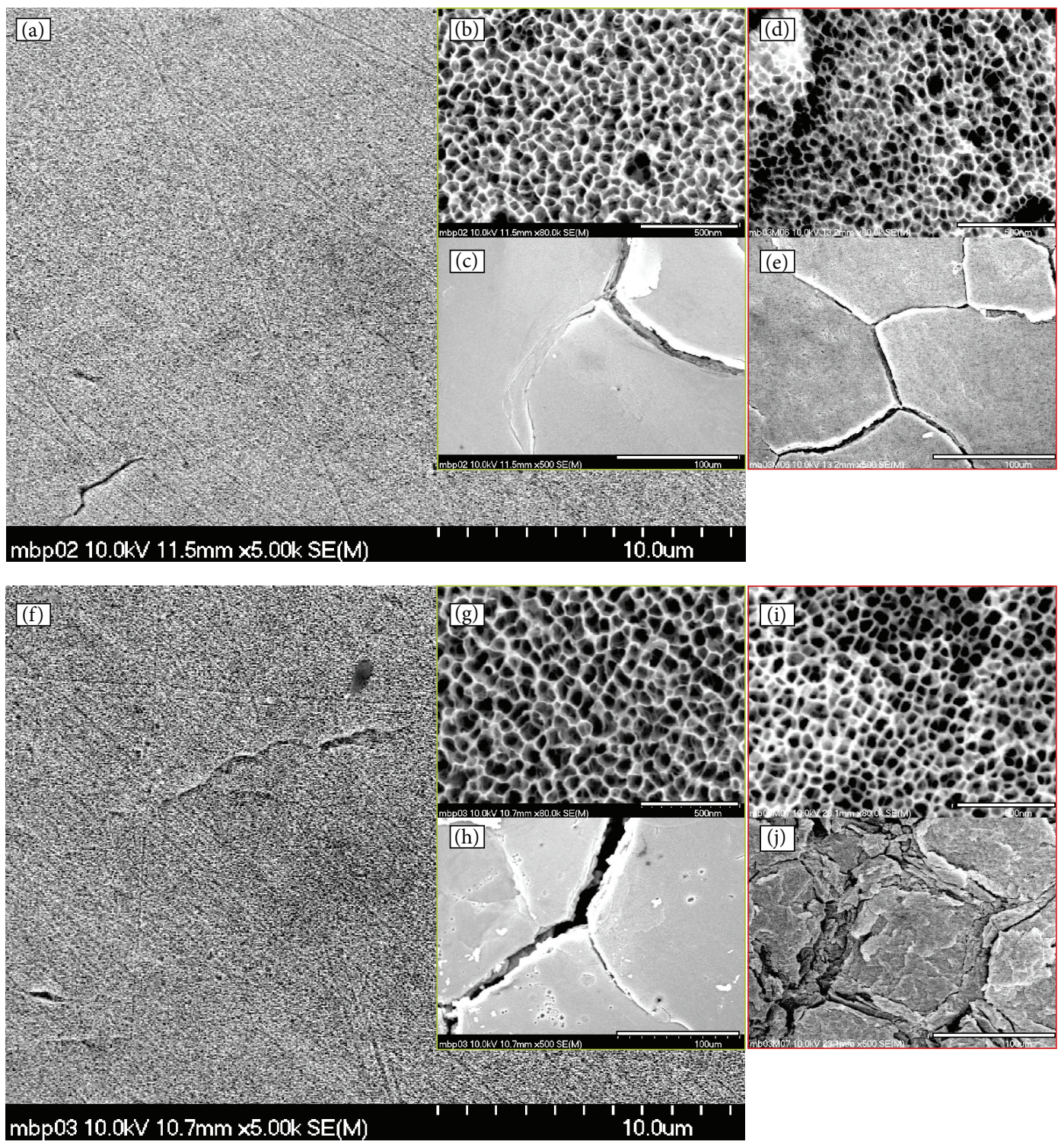

FIGURE 7: FE-SEM images of nanoporous tin oxides formed during $1800 \mathrm{~s}$ anodization of the high-purity (a-c, f-h) and low-purity (d-e, i-j) Sn foils in a $0.3 \mathrm{M}$ oxalic acid electrolyte at the potential of $6 \mathrm{~V}(\mathrm{a}-\mathrm{e})$ and $7 \mathrm{~V}(\mathrm{f}-\mathrm{j})$. Scale bars in inset images indicate $500 \mathrm{~nm}(\mathrm{~b}, \mathrm{~d}$, g, and i) and $100 \mu \mathrm{m}$ (c, e, h, and j).

properties of the oxide layer and determines its possible applications (e.g., can offer larger surface area in case when nanoporous oxide is used as a gas sensing material).

The evolution of the porous oxide layer morphology during anodizing of $\mathrm{Sn}$ wire was found to be very similar to that observed during anodization of Sn foils [35] and metallic Sn deposited on the Cu support [19]. Briefly, just after the beginning of anodization, a formation of the "outer" oxide layer with very small, irregular nanochannels or even with completely closed pores is observed. Further continued anodization results in an electric field-assisted dissolution of the oxide at the oxide/electrolyte interface (see Figures $9(\mathrm{a})-9(\mathrm{c})$ illustrating an increase in the average diameter of nanopores in the "outer" oxide layer with increasing anodizing duration) and in a formation of the "inner" oxide layer with a well-defined porous structure, being a result of vigorous gas evolution during anodization (see Figure 9(d)).

It should be noted that too long anodization, especially under severe anodizing conditions (higher anodizing potentials and elevated temperatures), can result in the formation of the partially destroyed oxide layer (compare Figures 9(e) and 9(f)). Further practical application of such kind of fragile and mechanically unstable materials could be strongly limited.

The dependence between degree of pore opening in the "outer" oxide layer and anodizing potential was also confirmed for oxide layers obtained during anodization of Sn wire. As can be seen in Figures 10(a) and 10(b), an increase in anodizing potential from $5 \mathrm{~V}$ (a) to $7 \mathrm{~V}$ (b) results in the formation of much more open nanochannels. On the 


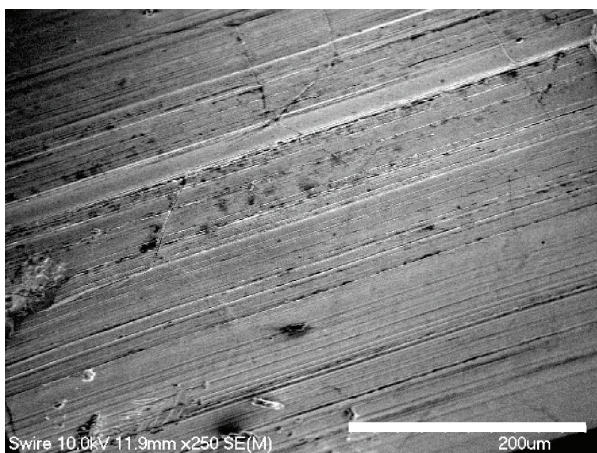

(a)

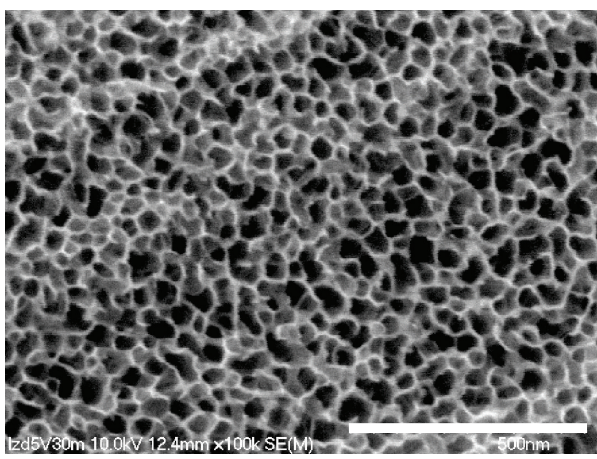

(c)

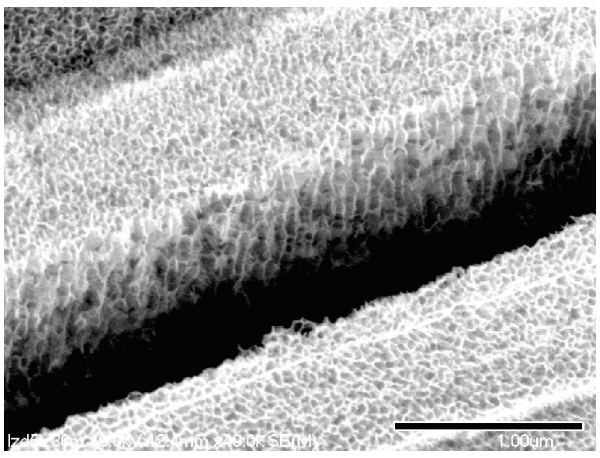

(e)

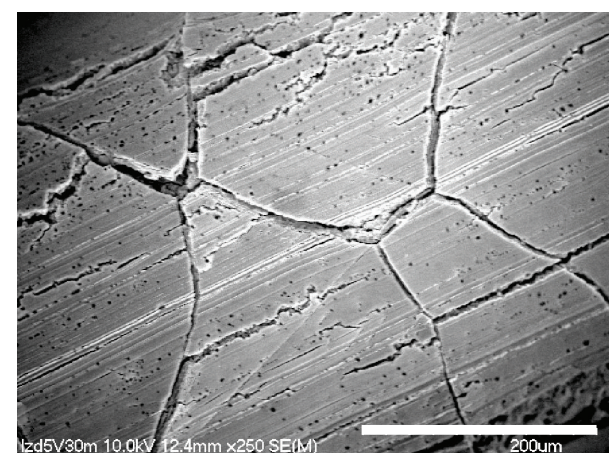

(b)

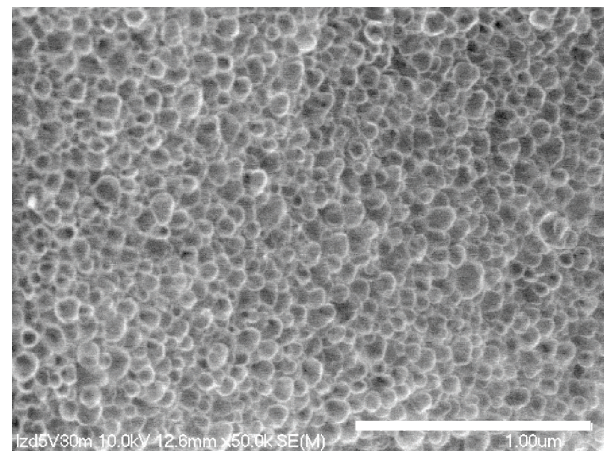

(d)

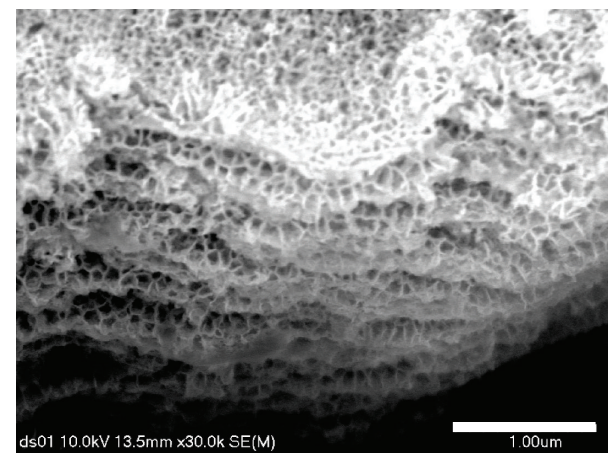

(f)

FIGURE 8: FE-SEM images of Sn wire before (a) and after $30 \mathrm{~min}$ of anodization in $0.3 \mathrm{M}$ at $5 \mathrm{~V}$ (b-f). Top (a, b), bottom (c), and cross-sectional $(e, f)$ views of the anodic oxide layer.

other hand, a further increase in the applied potential $(>9 \mathrm{~V})$ resulted in the initial formation of the passive layer and its further breakdown. As it was already mentioned, similar behavior was observed for anodization of low-purity $\mathrm{Sn}$ foils in the B-type cell and described in our recent paper [35].

\section{Conclusions}

The following conclusions can be drawn from this study:

(1) Nanoporous tin oxide layers can be easily formed on various Sn substrates including high- and lowpurity foils and wire via one-step cost-effective anodic oxidation in oxalic acid electrolyte.
(2) As-obtained oxide is amorphous and the atomic ratio of $\mathrm{Sn}: \mathrm{O}(1: 1)$ as well as dark color suggests that it is mainly composed of SnO. However, the exact nature and composition of such kind of the material are still uncertain.

(3) The typical structure of the nanoporous oxide film consists of the "outer" layer with less regular, often interconnected pores formed at early stages of anodic oxidation and the "inner" structure with more uniform and regular nanochannels formed as a result of vigorous oxygen evolution. An average pore diameter in the "outer" oxide layer increases with increasing anodizing potential. 


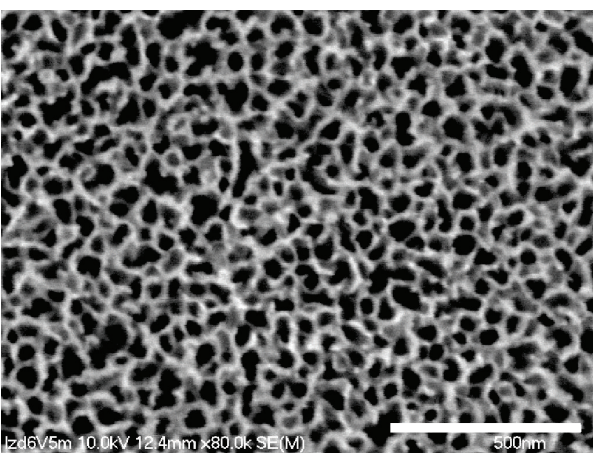

(a)

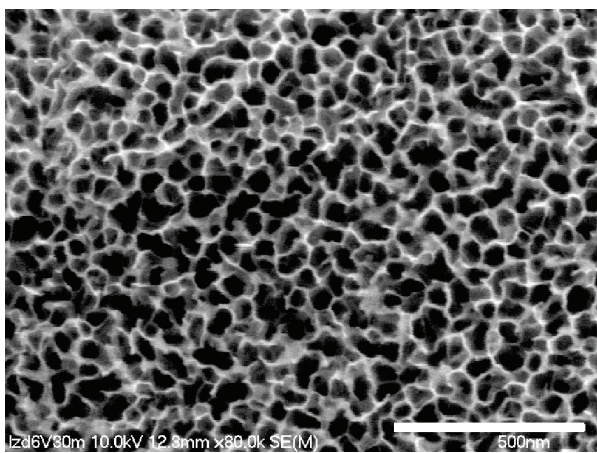

(c)

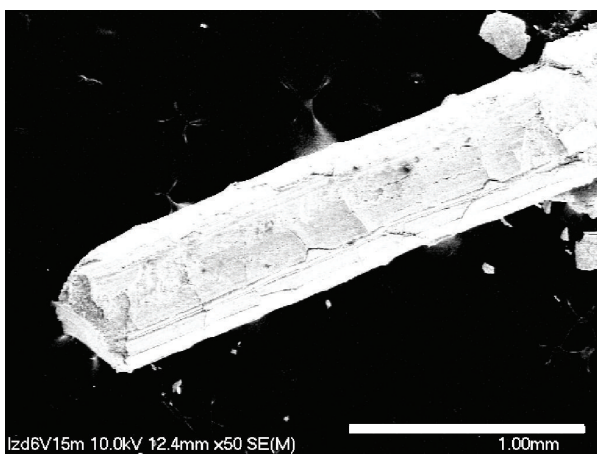

(e)

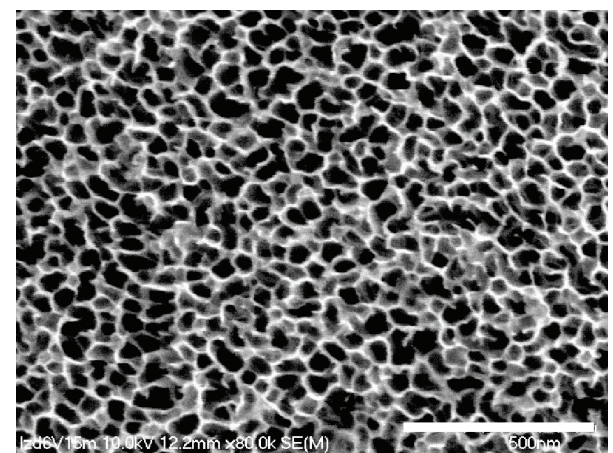

(b)

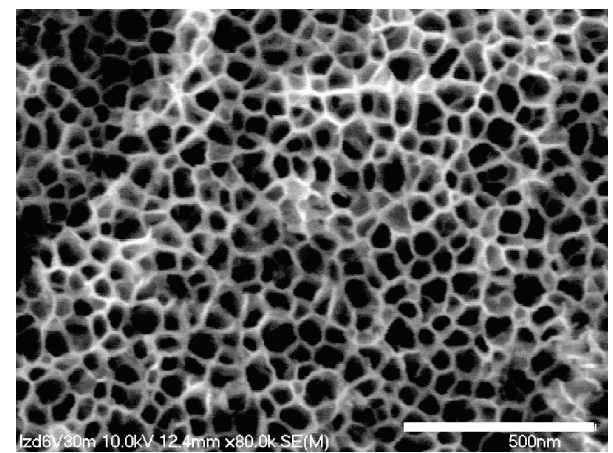

(d)

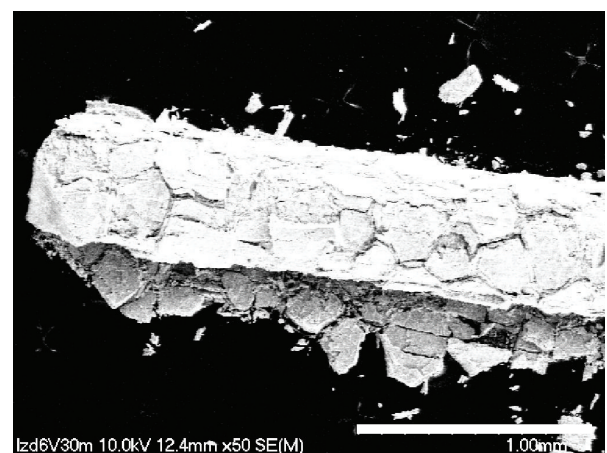

(f)

FIGURE 9: FE-SEM images of nanoporous tin oxide layers formed during one-step anodizing of Sn wire in $0.3 \mathrm{M}$ oxalic acid at $6 \mathrm{~V}$. The duration of anodization was $5 \mathrm{~min}$ (a), $15 \mathrm{~min}$ (b, e), and $30 \mathrm{~min}$ ((c): "outer" layer, (d): "inner" layer, and (f)).

(4) A strong linear relationship between the average current density and applied potential suggests that the process is limited by mass transfer in the electrolyte.

(5) No significant effect of substrate purity on the structure of anodic film was proved, except better uniformity of the oxides grown on high-purity $S n$.

We strongly believe that such nanoporous oxides could be excellent candidates for various applications including highperformance gas sensors, catalysts, and other electrochemical purposes.

\section{Conflict of Interests}

The authors declare that there is no conflict of interests regarding the publication of this paper.

\section{Acknowledgments}

The research was partially supported by the Polish Ministry of Science and High Education (Project Iuventus Plus no. IP2012 057372). The authors would like to acknowledge Dr. Katarzyna Hnida (Academic Centre for Materials and 


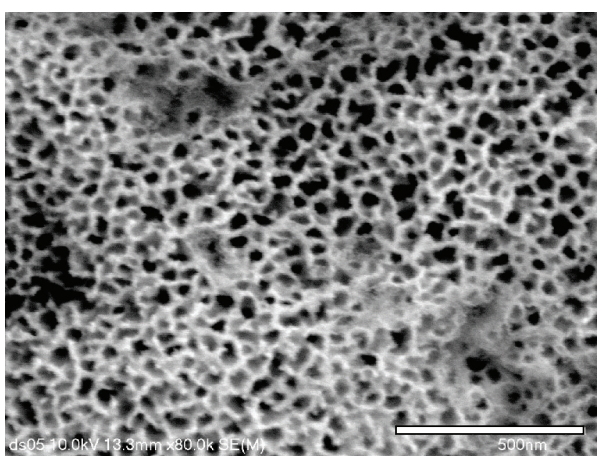

(a)

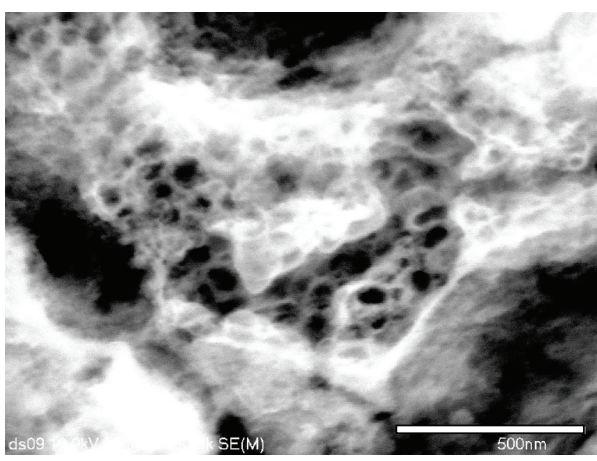

(c)

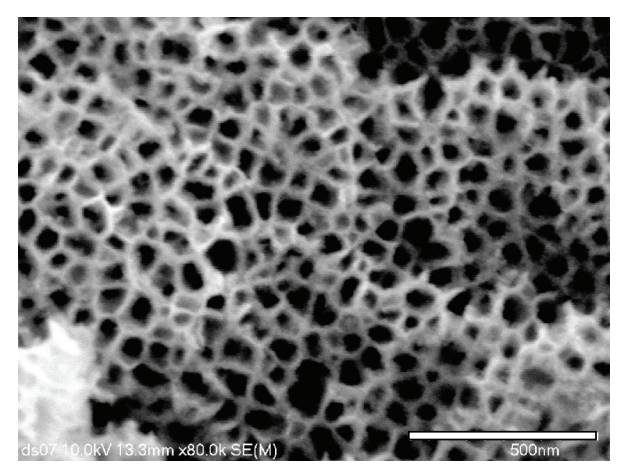

(b)

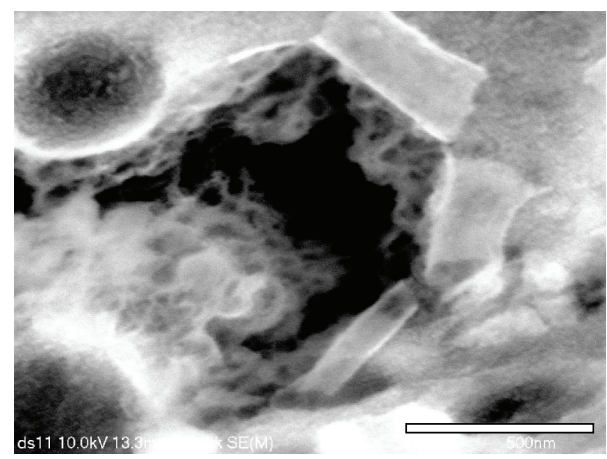

(d)

Figure 10: FE-SEM images of anodic tin oxides after $5 \mathrm{~min}$ of anodizing of Sn wire in $0.3 \mathrm{M}$ oxalic acid at $5 \mathrm{~V}$ (a), $7 \mathrm{~V}$ (b), $9 \mathrm{~V}$ (c), and $11 \mathrm{~V}$ (d).

Nanotechnology, AGH University of Science and Technology, Krakow, Poland) for XRD measurements and Dolores Stokłosa for the assistance in anodization of Sn wire samples. The SEM imaging was performed in the Laboratory of Field Emission Scanning Electron Microscopy and Microanalysis at the Institute of Geological Sciences, Jagiellonian University, Poland.

\section{References}

[1] G. Korotcenkov, "Metal oxides for solid-state gas sensors: what determines our choice?" Materials Science and Engineering B: Solid-State Materials for Advanced Technology, vol. 139, no. 1, pp. $1-23,2007$.

[2] T. A. Miller, S. D. Bakrania, C. Perez, and M. S. Wooldridge, "Nanostructured tin dioxide materials for gas sensor applications," in Functional Nanomaterials, K. E. Geckeler and E. Rosenberg, Eds., pp. 1-24, American Scientific, 2006.

[3] M. Batzill and U. Diebold, "The surface and materials science of tin oxide," Progress in Surface Science, vol. 79, no. 2-4, pp. 47-154, 2005.

[4] Y. Fukai, Y. Kondo, S. Mori, and E. Suzuki, "Highly efficient dyesensitized $\mathrm{SnO}_{2}$, solar cells having sufficient electron diffusion length," Electrochemistry Communications, vol. 9, no. 7, pp. 1439-1443, 2007.

[5] J. Chen, "Recent progress in advanced materials for lithium ion batteries," Materials, vol. 6, no. 1, pp. 156-183, 2013.
[6] H. Wang and A. L. Rogach, "Hierarchical $\mathrm{SnO}_{2}$ nanostructures: recent advances in design, synthesis, and applications," Chemistry of Materials, vol. 26, no. 1, pp. 123-133, 2014.

[7] S.-H. Nam and J.-H. Boo, "Rutile structured $\mathrm{SnO}_{2}$ nanowires synthesized with metal catalyst by thermal evaporation method," Journal of Nanoscience and Nanotechnology, vol. 12, no. 2, pp. 1559-1562, 2012.

[8] Y. Li, R. Peng, X. Xiu, X. Zheng, X. Zhang, and G. Zhai, "Growth of $\mathrm{SnO}_{2}$ nanoparticles via thermal evaporation method," Superlattices and Microstructures, vol. 50, no. 5, pp. 511-516, 2011.

[9] H.-C. Chiu and C.-S. Yeh, "Hydrothermal synthesis of $\mathrm{SnO}_{2}$ nanoparticles and their gas-sensing of alcohol," Journal of Physical Chemistry C, vol. 111, no. 20, pp. 7256-7259, 2007.

[10] X. Zhang, J. Liang, G. Gao et al., "The preparation of mesoporous $\mathrm{SnO}_{2}$ nanotubes by carbon nanofibers template and their lithium storage properties," Electrochimica Acta, vol. 98, pp. 263-267, 2013.

[11] Y. Wang, M. Aponte, N. Leon et al., "Synthesis and characterization of tin oxide microfibres electrospun from a simple precursor solution," Semiconductor Science and Technology, vol. 19, no. 8, pp. 1057-1060, 2004.

[12] D. Vasanth Raj, N. Ponpandian, D. Mangalaraj, and C. Viswanathan, "Electrochemical behavior of nanostructured $\mathrm{SnO}_{2}$ thin films in aqueous electrolyte solutions," Materials Science in Semiconductor Processing, vol. 26, no. 1, pp. 55-61, 2014.

[13] H.-C. Shin, J. Dong, and M. Liu, "Porous tin oxides prepared using an anodic oxidation process," Advanced Materials, vol. 16, no. 3, pp. 237-240, 2004. 
[14] W.-Q. Han, G. Zhou, L.-J. Wang, X.-Y. Sun, and J.-S. Li, "Fabrication of tin dioxide nanotubes by anodic oxidation," Journal of Inorganic Materials, vol. 22, no. 3, pp. 395-399, 2007.

[15] J.-H. Jeun and S.-H. Hong, "CuO-loaded nano-porous $\mathrm{SnO}_{2}$ films fabricated by anodic oxidation and RIE process and their gas sensing properties," Sensors and Actuators B: Chemical, vol. 151, no. 1, pp. 1-7, 2010.

[16] H. Chen, W. Zhu, X. Zhou, J. Zhu, L. Fan, and X. Chen, "Formation of porous $\mathrm{SnO}_{2}$ by anodic oxidation and their optical properties," Chemical Physics Letters, vol. 515, no. 4-6, pp. 269273, 2011.

[17] J.-W. Lee, S.-J. Park, W.-S. Choi, and H.-C. Shin, "Well-defined meso- to macro-porous film of tin oxides formed by an anodization process," Electrochimica Acta, vol. 56, no. 17, pp. 5919-5925, 2011.

[18] A. Yamaguchi, T. Iimura, K. Hotta, and N. Teramae, "Transparent nanoporous tin-oxide film electrode fabricated by anodization," Thin Solid Films, vol. 519, no. 8, pp. 2415-2420, 2011.

[19] L. Zaraska, N. Czopik, M. Bobruk, G. D. Sulka, J. Mech, and M. Jaskuła, "Synthesis of nanoporous tin oxide layers by electrochemical anodization," Electrochimica Acta, vol. 104, pp. 549-557, 2013.

[20] H. Cheng, S. Shu, Z. Lu et al., "Electrochemical fabrication and optical properties of porous tin oxide films with structural colors," Journal of Applied Physics, vol. 116, no. 15, Article ID 153511, 2014.

[21] M. Wang, Y. Liu, D. Xue, D. Zhang, and H. Yang, "Preparation of nanoporous tin oxide by electrochemical anodization in alkaline electrolytes," Electrochimica Acta, vol. 56, no. 24, pp. 8797-8801, 2011.

[22] M. Wang, H. Yang, and Y. Liu, "Current oscillations during potentiostatic anodization of tin in alkaline electrolytes," Electrochimica Acta, vol. 56, no. 20, pp. 7051-7057, 2011.

[23] J. J. Teh, G. H. Guai, X. Wang, K. C. Leong, C. M. Li, and P. Chen, "Nanoporous tin oxide photoelectrode prepared by electrochemical anodization in aqueous ammonia to improve performance of dye sensitized solar cell," Journal of Renewable and Sustainable Energy, vol. 5, no. 2, Article ID 023120, 2013.

[24] A. Palacios-Padrós, M. Altomare, A. Tighineanu et al., "Growth of ordered anodic $\mathrm{SnO}_{2}$ nanochannel layers and their use for $\mathrm{H}_{2}$ gas sensing," Journal of Materials Chemistry A, vol. 2, no. 4, pp. 915-920, 2014.

[25] A. Palacios-Padrós, M. Altomare, K. Lee, I. Díez-Pérez, F. Sanz, and P. Schmuki, "Controlled thermal annealing tunes the photoelectrochemical properties of nanochanneled tin-oxide structures," ChemElectroChem, vol. 1, no. 7, pp. 1133-1137, 2014.

[26] L. Zaraska, G. D. Sulka, and M. Jaskula, "Anodic alumina membranes with defined pore diameters and thicknesses obtained by adjusting the anodizing duration and pore opening/widening time," Journal of Solid State Electrochemistry, vol. 15, no. 11-12, pp. 2427-2436, 2011.

[27] G. D. Sulka, J. Kapusta-Kołodziej, A. Brzózka, and M. Jaskuła, "Anodic growth of $\mathrm{TiO}_{2}$ nanopore arrays at various temperatures," Electrochimica Acta, vol. 104, pp. 526-535, 2013.

[28] S. Feng, Y. Tang, and T. Xiao, "Anodization, precursor route to flowerlike patterns composed of nanoporous tin oxide nanostrips on tin substrate," Journal of Physical Chemistry C, vol. 113, no. 12, pp. 4809-4813, 2009.

[29] J.-H. Jeun, H.-S. Ryu, and S.-H. Hong, "Nanoporous $\mathrm{SnO}_{2}$ film gas sensor formed by anodic oxidation," Journal of the Electrochemical Society, vol. 156, no. 9, pp. J263-J266, 2009.
[30] J.-W. Lee, S.-J. Park, and H.-C. Shin, "Electrochemical characterization of anodic tin oxides with nano-porous structure," Korean Journal of Materials Research, vol. 21, no. 1, pp. 21-27, 2011.

[31] G. F. Ortiz, P. Lavela, P. Knauth, T. Djenizian, R. Alcntara, and J. L. Tirado, "Tin-based composite materials fabricated by anodic oxidation for the negative electrode of Li-ion batteries," Journal of the Electrochemical Society, vol. 158, no. 10, pp. A1094-A1099, 2011.

[32] S.-J. Park and H.-C. Shin, "Fabrication of nano-channeled tin oxide film electrode and evaluation of its electrochemical properties," Korean Journal of Materials Research, vol. 22, no. 1, pp. 1-7, 2012.

[33] W. Jiang, W. Zeng, Z. Ma, Y. Pan, J. Lin, and C. Lu, "Advanced amorphous nanoporous stannous oxide composite with carbon nanotubes as anode materials for lithium-ion batteries," RSC Advances, vol. 4, no. 78, pp. 41281-41286, 2014.

[34] D. V. Shinde, D. Y. Lee, S. A. Patil et al., "Anodically fabricated self-organized nanoporous tin oxide film as a supercapacitor electrode material," RSC Advances, vol. 3, no. 24, pp. 9431-9435, 2013.

[35] L. Zaraska, M. Bobruk, M. Jaskuła, and G. D. Sulka, "Growth and complex characterization of nanoporous oxide layers on metallic tin during one-step anodic oxidation in oxalic acid at room temperature," Applied Surface Science, vol. 351, pp. 10341042, 2015.

[36] S. Ono and H. Asoh, "Fabrication of self-organized nanoporous tin oxide by anodization," in Proceedings of the 211th ECS Meeting, Abstract \#1331, Chicago, Ill, USA, May 2007. 

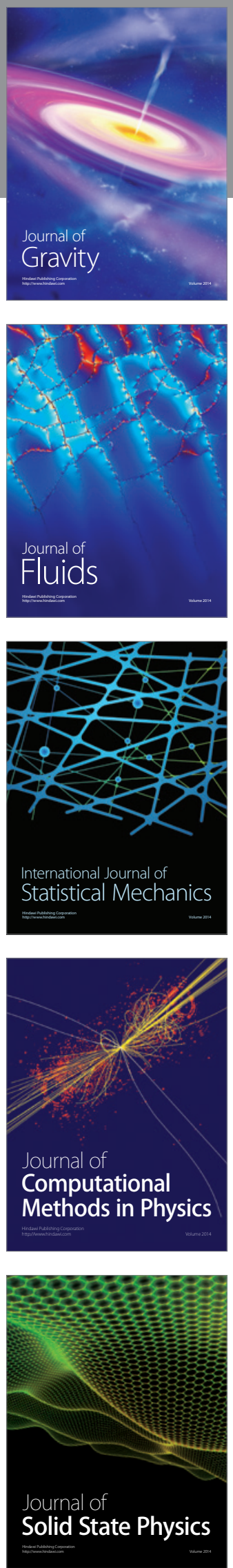

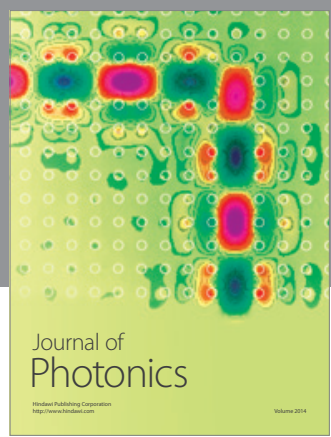

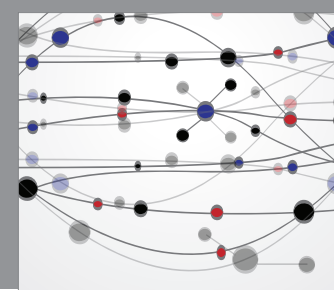

The Scientific World Journal

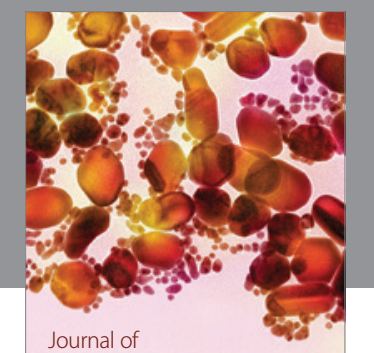

Soft Matter
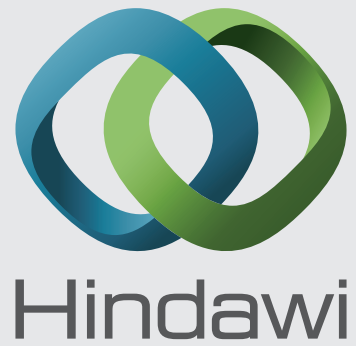

Submit your manuscripts at

http://www.hindawi.com
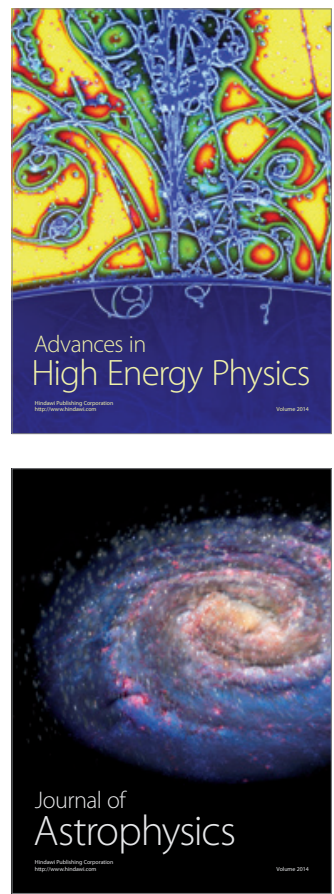
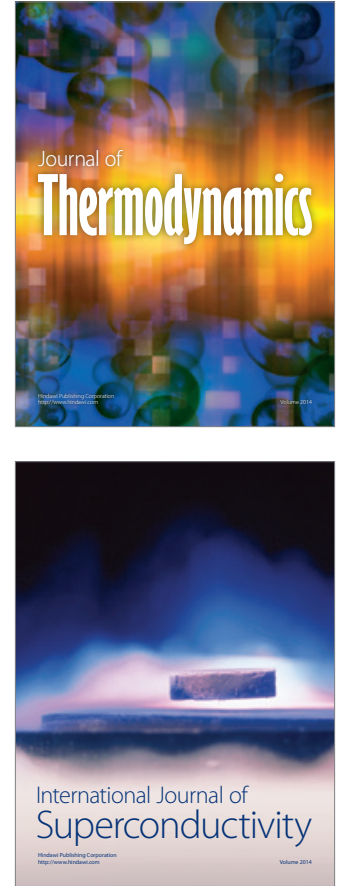
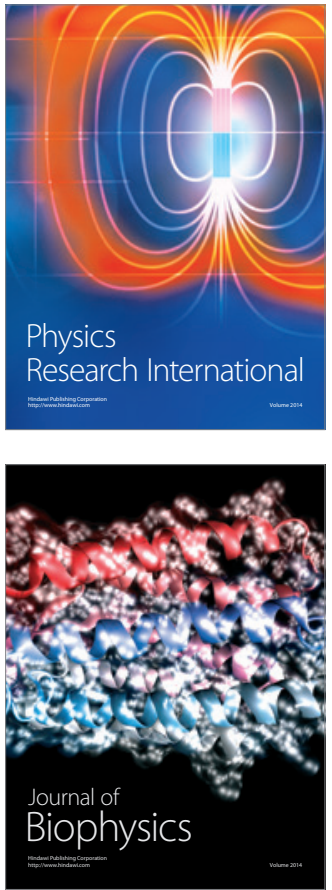
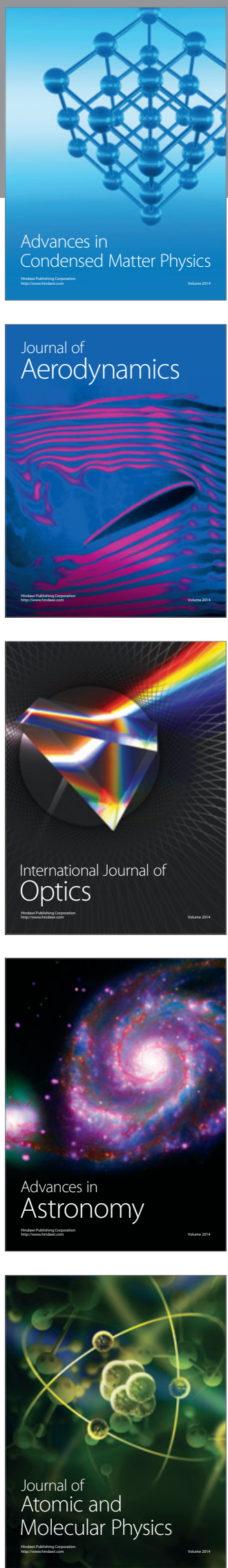